\title{
Evaluation of Upper Extremity Deep Vein Thrombosis Risk Factors and Management
}

\author{
Heena Sheth ${ }^{1 *}$, Rahim Remtulla ${ }^{2}$, Abuzar Moradi ${ }^{2}$ and Roy Smith ${ }^{2}$ \\ ${ }^{1}$ Department of Medicine, University of Pittsburgh School of Medicine \\ ${ }^{2}$ Division of Hematology Oncology, University of Pittsburgh Medical Center, Pittsburgh, PA, USA
}

"Corresponding Author: Dr. Heena Sheth, Montefiore University Hospital, 3404 Fifth Avenue, Suite G100, Pittsburgh, PA 15213, USA, E-mail: hss2@pitt.edu

Received: 27 January 2018; Accepted: 08 February 2018; Published: 15 February 2018

\begin{abstract}
Background: Upper extremity deep venous thrombosis (UEDVT) incidence and complications are increasing. We evaluated the risk factors and management for patients diagnosed with an UEDVT.

Methods: All patients with an UEDVT in 2014 were evaluated for demographics, thrombosis characteristics, risk factors, PADUA risk score, outcomes and management.

Results: Ninety UEDVT patients with mean age 57 years, 54.4\% maleS, 31\% obese (BMI $\geq 30 \mathrm{~kg} / \mathrm{m} 2$ ), 26.7\% prior VTE, 31.1\% readmitted within 30 days. Median length of stay was 14 versus hospital's 5-7days. 87.8\% (n=79) were high risk on admission, $69.6 \%$ on anticoagulation when diagnosed. Risk factors: Immobility for 3 days 75 (83.3\%), Age $<70$ years $70(77.8 \%)$, central venous catheters 70 (77.8\%), male gender $49(54.4 \%)$, orthopedic trauma / surgery $40(44.4 \%)$, infection/inflammation $33(36.7 \%)$, obesity 28 (31.1\%), history of prior VTE 24 (26.7\%) and malignancy $16(17.6 \%)$. Hypercoagulability, hormone therapy and pacemaker were noted in $<10 \%$ of patients. $87.1 \%$ of catheters were PICC lines majority being multi-lumen (98.4\%). Indications for catheters were $80.1 \%$ medication administration, 7.4\% TPN, 3.3\% ICU resuscitation, 3.3\% dialysis/pheresis. Dedicated IV/PICC team placed $55.8 \%$ catheters and $10 \%$ had required greater than one attempt. $62.2 \%$ UEDVTs were right-sided with, $84.4 \%$ proximal and $15.6 \%$ distal DVTs. $87 \%(n=60)$ catheters were extracted after diagnosis. Therapeutic anticoagulation at discharge was prescribed for only $81.6 \%$ of patients with proximal UEDVTs (recommended) and $50 \%$ for distal UEDVTs (not recommended). Lower extremity ultrasonography was performed in only $34.4 \%$ of patients. $6.7 \%(\mathrm{n}=6)$ had LEDVT, $3.3 \%(\mathrm{n}=3) \mathrm{PE}$, and $1.1 \%(\mathrm{n}=1)$ died.
\end{abstract}

Conclusions: UEDVT most common risk factors $(>50 \%)$ were decreased mobility, younger age, central venous catheters, males. UEDVT management was suboptimal and warrants standardization. 
Keywords: Upper extremity; Deep vein thrombosis; Anticoagulation; Risk factors

\section{Upper Extremity DVT}

The reporting, understanding, and awareness of the differences and similarities of upper extremity deep venous thrombosis (UEDVT) as compared to lower extremity DVTs have all greatly increased in recent years [1] UEDVT incidence rates have risen with increasing use of intravenous devices particularly central venous catheters (CVC) including peripherally inserted central catheters (PICC) [2]. The incidence of UEDVT is reported as approximately $10 \%$ of all DVTs and varies from 4 to $14 \%$ in patients with CVCs [3, 4]. Complications of UEDVT were also previously believed to be rare and insignificant compared to LEDVT. However, recent studies have demonstrated that UEDVT are associated with significant morbidity (pulmonary embolism (PE), post thrombotic syndrome) and even mortality [5-8].

Compared to LEDVT, UEDVT patients tend to be younger, weigh less, have underlying malignancies, and are less commonly associated with thrombophilia [8,9]. The risk factors for UEDVT are well established [7]. Primary UEDVTs occur in about one quarter to one third of cases while the majority are secondary in nature associated with risk factors such as intravenous catheters and devices, surgery/trauma, malignancy, estrogen related (pregnancy and oral contraceptives) or due to thrombophilia [8-11]. Primary UEDVTs are frequently associated with the venous thoracic outlet syndrome [12] due to repetitive micro trauma to the venous vasculature or effort related (PagetSchroetter syndrome) $[13,14]$ while idiopathic primary UEDVTs constitute a small proportion. Known CVC/PICC characteristics and related risk factors to include use of multi-lumen catheters, number of insertion attempts, duration of use [15], the administration of chemotherapy or parenteral infusion, [16] left sided location, [17, 18] insertion site [19], catheter material, prior placement of CVC/PICC, and catheter associated infections have been well documented and have led to methods to reduce the occurrence $\mathrm{CVC} / \mathrm{PICC}$-associated venous thrombosis. Obesity and Immobility are established risk factors for LEDVT but they are not considered significant for UEDVT. However, Stone et al., recently demonstrated that immobility within 30 days was a common risk factor for the UEDVT [20].

Patients diagnosed with an UEDVT often have a longer duration of hospitalization and incur increased health related costs. Thus, it is imperative to identify patients at high risk for developing UEDVT to improve patient outcomes and reduce costs [19]. Furthermore, appropriate management of newly diagnosed UEDVT in the setting of central catheters which may or may not be amenable or appropriate for removal is essential in preventing known complications. Regarding management, Chest guidelines for the management of venous thromboses lists a grade $2 \mathrm{C}$ recommendation of a minimum of three months of anticoagulation for the proximal UEDVTs (defined as within the axillary vein or more proximal) [21]. Furthermore, these guidelines also describe the indications of catheter removal in the setting of newly diagnosed UEDVT. Patients with an UEDVT, especially in the recurrent setting, should be evaluated for lower extremity DVT and associated thrombophilia. Currently, at our institution there is no standardized protocol for UEDVT diagnosis and management. Our study was initiated following the root cause analysis of an upper extremity deep venous thrombotic event which identified a fatal pulmonary embolus in a patient 
who was not adequately anticoagulated. This case prompted a thorough evaluation for risk factors and management of UEDVT to determine the current practice and compliance with guidelines and inform the specific knowledge deficits to be addressed in developing educational programs and standardized management protocols.

\section{Methods}

Upper extremity deep venous thrombosis characteristics, risk factors, associated complications, and management at an urban tertiary care hospital were retrospectively reviewed from the health system's electronic medical record. Upper extremity deep venous thrombosis cases that occurred in calendar year 2014 were identified from the institution's surveillance database of the hospital acquired conditions. The venous thromboembolism (VTE) cases (DVT and PE) were routinely collected for surveillance by diagnosis related group query for DVT from the electronic medical records. The study team acquired the UEDVT patient list with basic information including demographics, admitting service, hospitalization information (length of stay, readmissions), VTE information (date of event and anticoagulation at the time of event) from the surveillance database. The study team verified this information and collected additional variables to include body mass index (BMI), UEDVT location (proximal or distal vein), laterality, indication for insertion, and associated lower extremity DVT, PE and death. PADUA score, which is an established scoring system to assess risk of VTE among hospitalized medical patients, was also formulated. The risk score assigns varying levels of points for the following risk factors: active cancer ( 3 points), previous VTE ( 3 points), decreased mobility ( 3 points), thrombophilia ( 2 points), previous trauma or surgery within that last month (1 point), age $\geq 70$ (1 point), heart and/or respiratory failure (1 point), ischemic stroke or acute myocardial infarction (1 point), acute rheumatologic disorder and/or acute infection (1 point), obesity (1 point), and current hormonal therapy (1 point). Patients are considered at high risk for DVT if they have four or more points which numerous hospital systems utilize to determine appropriate methods of prophylactic anticoagulation. The details on intravenous devices and catheters were collected such as type of device, qualification of provider inserting the device, duration of catheter use, UEDVT preceded by CVC/PICC infection or bacteremia, and number of catheter lumens. Finally, practice patterns were characterized by identifying anticoagulation details at the time of UEDVT (i.e. type of anticoagulant, prophylactic or therapeutic dosing), management at the time of discharge, rate of obtaining lower extremity venous duplex Doppler ultrasonography, and if catheters were extracted after the diagnoses of UEDVT.

To minimize the variation in data collection each study team member who reviewed the medical records was instructed in detail regarding definitions and criteria for each variable. The project was approved as a quality improvement project from the institutional quality council and did not require full institutional review board approval.

\subsection{Statistical analysis}

Descriptive analysis was used to describe the study population. The risk factors are presented as percentages and management variables are also described in percentage form. Depending upon the type of variable and distribution 
the mean, median, range and standard deviation are reported. All analysis was conducted using STATA version 9; Stata Corporation Inc., TX, USA.

\section{Results}

\subsection{Patient characteristics and risk factors}

Ninety patients with venous duplex Doppler ultrasonography confirmed UEDVTs were identified over a one year period (2014) with notable characteristics of mean age 57 years (SD 15.9, range 18-91), 54.4\% male, 31\% with BMI $\geq 30 \mathrm{~kg} / \mathrm{m} 2,26.7 \%$ with prior history of VTE and $31.1 \%$ hospitalized within the preceding 30 days. Median length of stay was 14 days compared to the hospital's average length of stay of 5-7days. 52\% of patients were admitted to the ICU with median length of stay of 9 days. Mean platelet levels were 234,000/ $\mu \mathrm{L}$ (SD 129,000/ $\mu \mathrm{L}$, range 41,000$582,000 / \mu \mathrm{L}$ ) of which $54.4 \%$ of patients were within $150,000 / \mu \mathrm{L}$ and $300,000 / \mu \mathrm{L}$.

PADUA risk scores were calculated on all patients with mean score of 6.3 (SD 2.5, range 0-11) with 87.8\% (n=79) considered high risk for VTE with score $\geq 4$. Of those considered high risk for VTE, $69.6 \%$ were on therapeutic or prophylactic anticoagulation at the time of diagnosis. The most common risk factors were decreased mobility in $83.3 \%(\mathrm{~N}=75)$, and catheter /port placement in $77.8 \%$ patients $(\mathrm{N}=70)$. The types of devices were (5 CVC, 61 PICCs, 2 dialysis catheters, 1 port, and 1 pharesis catheter). Additional risk factors are described in Table 2.

Of the 70 catheters or ports placed, $87.1 \%$ were PICC lines of which $98.4 \%$ were double or triple lumen. Indications for catheter placement were $81.4 \%$ medication administration, $7.1 \%$ TPN, $7.1 \%$ ICU resuscitation, $4.3 \%$ dialysis/pheresis. $55.8 \%$ of catheters were placed by dedicated IV/PICC line team and 10\% required greater than one attempt at time of placement. $12.2 \%$ of all patients were bacteremic with $6.7 \%$ due to a catheter associated infection.

\subsection{VTE Characteristics}

Location of the most proximal aspect of the UEDVT was identified by venous duplex Doppler ultrasonography in all ninety patients with $62.2 \%$ within the right upper extremity. In total $84.4 \%$ UEDVT were in proximal veins (, $38.9 \%$ within the axillary vein, $27.8 \%$ within subclavian vein, and $16.7 \%$ within internal jugular vein), while $15.6 \%$ were distal within the brachial veins.

\subsection{Management and outcomes}

Regarding management, $87 \% \%(\mathrm{n}=60)$ of all catheters were extracted after diagnosis. $81.6 \%$ of patients with proximal DVTs defined as axillary, subclavian, internal jugular veins were discharged on therapeutic anticoagulation. $50 \%$ of patients with distal DVTs (brachial vein or more distal) were discharged on therapeutic anticoagulation. Lower extremity venous duplex Doppler ultrasonography was performed in $34.4 \%$ of patients. There was $6.7 \%, 3.3 \%$ and $1.1 \%$ rate of lower extremity DVT, PE, and death, respectively. 


\section{Discussion}

Our evaluation demonstrated that UEDVTs were occurring more frequently in patients who had experienced immobility 3 days prior to the occurrence of UEDVT, and in young age ( $<70$ years) patients. Orthopedic trauma/surgery, infection inflammation, obesity (BMI>30) and malignancy were also relatively common. A small proportion of patients with UEDVT also had evidence of a thrombophilia (6.7\%). These findings are in line with previously reported risk factors for UEDVT patient characteristics $[8,9,20]$.

The majority of UEDVTs occurred in patients with use of CVC's (62\%), an expected finding consistent with prior reports [2, 3, 17-21]. In our evaluation, the catheter associated UEDVTs occurred more frequently in multi-lumen PICC lines inserted in the axillary vein or more proximal position for the purpose of medication administration . Our study did not demonstrate high proportion of catheters use for TPN, ICU resuscitation, or dialysis/pheresis. Overall, rates of central catheter associated infections at our institution are very low which was reflective in our population. There were very few lines associated with poor insertion techniques as only $10 \%$ lines had required more than one attempt at insertion, a reflection of a dedicated service proficient in CVC/PICC placement.

Immobility is not a recognized risk for UEDVT and its contribution to development of the DVT in UE is not studied. However, our study found $83 \%$ of patients were immobile within 3 days. This warrants importance of mobilizing patients in the hospital focusing on upper extremities as well and further studies to evaluate immobility as risk for the UEDVT. It may be contributing risk to other factors such as obesity, malignancies and inflammation/infection.

Our study findings demonstrate suboptimal management of UEDVT. Only $70 \%$ of patients considered high risk for venous thromboembolism (PADUA score $\geq 4$ ) were on prophylactic or therapeutic anticoagulation at the time of developing an UEDVT which demonstrates the importance of identifying and adequately anticoagulating these patients. According to the published guidelines, [22-24] patients with proximal UEDVTs should be anticoagulated for at least 3 months or for the duration of catheter placement, whichever is longer; while distal UEDVT generally do not require therapeutic anticoagulation. In our population, $80 \%$ of patients with proximal UEDVT were appropriately anticoagulated; however, 50\% of those with distal UEDVT were inappropriately placed on anticoagulation increasing risks of bleeding complications. Patients diagnosed with an UEDVT should have a lower extremity ultrasonography performed; however, only $34 \%$ of our population was evaluated in such manner. Lastly, $87 \%(n=60)$ of all catheters were extracted after diagnosis of the UEDVT. CHEST guidelines recommend (grade 2C) that catheters should not be removed if they are functional with an ongoing need for use [22, 25]. These management practices at our institute are suboptimal and warrant need for management protocols and provider education.

Our study is limited by its retrospective nature and potential for inaccurate and missing data. There was no comparative control arm thus we were unable to determine the attribution of risk. However, this was a quality 
improvement study to define our UEDVT patient population and practice patterns to find opportunities for education and improvement.

In summary, UEDVT were associated with risk factors such as immobility, male patient, younger age, PICC lines, multiple lumens and proximal (axillary being most common) insertion site, malignancies, obesity and infection/inflammation. Insertion process inadequacy and line infections were not found in our study likely due to proficiency of dedicated services. UEDVT management was not optimal and warrants protocol development and education which are likely applicable to many other institutions.

\section{References}

1. Horattas MC, Wright DJ, Fenton AH, et al. Changing concepts of deep venous thrombosis of the upper extremity. Report of a series and review of the literature. Surgery 1988;104:561-567

2. Abdullah, BJ, Mohammad, N, Sangkar, JV, Abd Aziz, YF, Gan, GG, Goh, KY, Benedict, I. Incidence of Upper Limb Venous Thrombosis Associated with Peripherally Inserted Central Catheters. Brit J Rad. 2005; 78:931, 596-600.

3. Joffe HV, Kucher N, Tapson VF, Goldhaber SZ. Upper-extremity deep vein thrombosis: a prospective registry of 592 patients. Circulation 2004;110:1605-11.

4. Isma N, Svensson PJ, Gottsäter A, Lindblad B. Upper extremity deep venousthrombosis in the populationbasedMalmö Thrombophilia Study (MATS): epidemiology, risk factors, recurrence risk, and mortality. Thromb Res 2010;125(6): e335-e338.

5. Hingorani A, Ascher E, Lorenson E, et al. Upper extremity deep venous thrombosis and its impact on morbidity and mortality rates in a hospital-based population. J Vasc Surg. 1997; 26: 853-860.

6. Muñoz FJ, Mismetti P, Poggio R, et al. Clinical outcome of patients with upper extremity deep vein thrombosis: results from the RIETE Registry. Chest 2008;133:143-8.

7. Kucher, N. Deep-Vein Thrombosis of the Upper Extremities. N Engl J Med 2011; 364:861-869.

8. Azi 1. Joffe HV, Kucher N, Tapson VF, Goldhaber SZ. Upper-extremity deep vein thrombosis: a prospective registry of 592 patients. Circulation 2004;110:1605-11.

9. Muñoz FJ, Mismetti P, Poggio R, et al. Clinical outcome of patients with upper extremity deep vein thrombosis: results from the RIETE Registry. Chest 2008;133:143-8.

10. Heron E, Lozinguez O, Alhenc-Gelas M, et al. Hypercoagulable states in primary upper-extremity deep vein thrombosis. Arch Intern Med. 2000; 160:382-386.

11. Martinelli I, Cattaneo M, Panzeri D, et al. Risk factors for deep venous thrombosis of the upper extremities. Ann Intern Med. 1997; 126: 707-711.

12. Isma N, Svensson PJ, Gottsäter A, Lindblad B. Upper extremity deep venous thrombosis in the populationbased Malmö Thrombophilia Study (MATS): epidemiology, risk factors, recurrence risk, and mortality. Thromb Res 2010;125(6): e335-e338.

13. Illig KA, Doyle AJ. A comprehensive review of Paget-Schroetter syndrome. J Vasc Surg 2010;51:1538-47. 
14. Linnemann B, Meister Fz S, Straehley CJ, Whelan TJ Jr. Effort-related axillosubclavian vein thrombosis:a new theory of pathogenesis and a plea for direct surgical intervention. Am J Surg 1986;152:57-61.

15. Baglin TP, Boughton BJ. Central venous thrombosis due to bolus injections of antileukaemic chemotherapy. Br J Haematol 1986;63:606-7.

16. Fabri PJ, Mirtallo JM, Ebbert ML, Kudsk KA, Powell C, Ruberg RL. Clinical effect of nonthrombotic total parenteral nutrition catheters. JPEN J Parenter Enteral Nutr 1984;8:705-7.

17. Marnejon T, Angelo D, Abu Abdou A, Gemmel D. Risk factors for upper extremity venous thrombosis associated with peripherally inserted central venous catheters. J Vasc Access. 2012 Apr-Jun;13(2):231-8. doi: 10.5301/jva.5000039.

18. Van Rooden CJ, Tesselaar MET, Osanto S, Rosendaal FR, Huisman MV. Deep vein thrombosis associated with central venous catheters - a review. J Thromb Haemost 2005; 3: 2409-19.

19. Jean-Jacques Parienti, Nicolas Mongardon, Bruno Mégarbane, Jean-Paul Mira, Pierre Kalfon, Antoine Gros, Sophie Marqué, et. al. Intravascular Complications of Central Venous Catheterization by Insertion Site. N Engl J Med 2015; 373:1220-1229. DOI: 10.1056/NEJMoa1500964

20. Rebecca H. Stone, Adam P. Bress, Edith A. Nutescu, and Nancy L. Shapiro. Upper-Extremity Deep-Vein Thrombosis: A Retrospective Cohort Evaluation of Thrombotic Risk Factors at a University Teaching Hospital Antithrombosis Clinic. Annals of Pharmacotherapy 2016, Vol. 50(8) 637-644

21. DOI: $10.1177 / 1060028016649601$

22. Kearon C, Akl EA, Ornelas J, et al. Antithrombotic therapy for VTE disease: chest guideline and expert panel report. Chest. 2016; 149(2):293-294

23. Barbar S, Noventa F, Rossetto V, Ferrari A, Brandolin B, Perlati M, De Bon E, Tormene D, Pagnan A, Prandoni P. A risk assessment model for the identification of hospitalized medical patients at risk for venous thromboembolism: the Padua Prediction Score. J Thromb Haemost 2010; 8: 2450-7.

24. Streiff, MD, et al. Guidance for the Treatment of Deep Vein Thrombosis and Pulmonary Embolism. J Thromb Thrombolysis 2016; 41:32-67.

25. Bleker SM, Van E N, Kleinjan A, B€uller HR, Kamphuisen PW, Aggarwal A, Beyer-Westendorf J, Camporese G, et. al. Current Management Strategies and Long-Term Clinical Outcomes of Upper Extremity Venous Thrombosis. J Thromb Haemost 2016; 14: 1-9.

26. Rathbun, SW, Stoner, JA, Whitsett, TL. Treatment of Upper-Extremity Deep Vein Thrombosis. J Thromb Haemost 2011; 9: 1924-1930.

Citation: Heena Sheth, Rahim Remtulla, Abuzar Moradi, Roy Smith. Evaluation of Upper Extremity Deep Vein Thrombosis Risk Factors and Management. Journal of Cancer Research 2 (2018): 18-24.

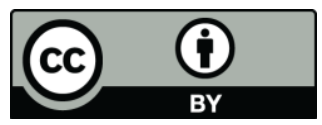

This article is an open access article distributed under the terms and conditions of the Creative Commons Attribution (CC-BY) license 4.0 